Classification

Physics Abstracts

$61.80 \mathrm{~F}-34.80 \mathrm{~B}-61.40 \mathrm{~K}$

\title{
Dégradation des monocristaux de polyéthylène en microscopie électronique entre 1 et 2,5 MV. I. Etude à température ambiante
}

\author{
A. Boudet et C. Roucau \\ Laboratoire d'Optique Electronique du C.N.R.S., 29, rue Jeanne Marvig, B.P. 4347, 31055 Toulouse Cedex, France
}

(Reçu le 16 janvier 1985, révisé le 15 avril, accepté le 24 avril 1985)

\begin{abstract}
Résumé. - On a pu mesurer la dose critique d'électrons qui détruit la cristallinité des monocristaux de polyéthylène, pour des tensions d'accélération entre 1 et 2,5 MV. Dans cette gamme d'énergie, la dose critique reste sensiblement constante et 3 fois plus grande qu'à $100 \mathrm{kV}$. On peut rendre compte de cette loi en calculant le pouvoir d'arrêt des électrons par la formule de Bethe corrigée pour tenir compte de la canalisation. On obtient alors une énergie critique de $490 \mathrm{eV} / \mathrm{nm}^{3}$ environ. La canalisation est plus importante pour les basses tensions, pour des épaisseurs moyennes $(20 \mathrm{~nm})$, et pour certaines orientations.
\end{abstract}

\begin{abstract}
The critical dose of electrons that destroys the crystallinity of polyethylene single crystals was measured for accelerating voltages between 1 and $2.5 \mathrm{MV}$. In this range, the critical dose remains nearly constant and is 3 times higher than at $100 \mathrm{kV}$. These results are fitted by a Bethe law corrected to take channelling into account. As a consequence, a critical energy of about $490 \mathrm{eV} / \mathrm{nm}^{3}$ is found. The channelling is more pronounced at lower voltages, at thicknesses of $20 \mathrm{~nm}$ and for particular orientations.
\end{abstract}

\section{Introduction.}

On sait que les monocristaux de polyéthylène, comme tous les cristaux organiques, s'altèrent lorsqu'ils sont bombardés par le faisceau d'un microscope électronique $[1,2]$.

L'observation du diagramme de diffraction 'électronique montre que les faisceaux diffractés s'affaiblissent puis disparaissent, traduisant la perte complète de la cristallinité pour une valeur $D_{\mathrm{c}}$ de la dose d'électrons reçue par l'échantillon. Cette dose n'est certes pas définie avec une grande précision et nous en discuterons la valeur. En même temps le contraste de l'image en fond noir s'atténue [3-5]. Or cette dégradation limite la résolution théorique que l'on peut espérer, qui est proportionnelle à $\left(D_{\mathrm{c}}\right)^{-1 / 2}[6]$. On a donc cherché à améliorer cette résolution en augmentant la dose critique par l'emploi des basses températures et des hautes tensions d'accélération des électrons, et en utilisant des doses minimales pour l'observation $[7,8]$.

Notre étude comporte 2 parties. Dans ce premier article, nous cherchons à savoir expérimentalement si l'emploi de tensions d'accélération comprises entre 1 et 2,5 MV peut apporter une amélioration. La deuxième partie est consacrée à l'influence des basses températures à ces mêmes tensions [9].
L'amélioration due à l'emploi de tensions jusqu'à $1 \mathrm{MV}$ a été testée plusieurs fois sur des monocristaux de polyéthylène [10-12]. On a constaté une nette augmentation de la dose critique qui est multipliée par un facteur 2 à 4 entre 100 et $1000 \mathrm{kV}$.

Le modèle généralement admis pour expliquer cette augmentation est le suivant : la destruction de la cristallinité résulte des pertes d'énergies dues aux chocs inélastiques des électrons sur les atomes. On peut calculer le pouvoir d'arrêt $\mathrm{d} E / \mathrm{d} x$ (perte par unité de longueur parcourue) grâce à la formule de Bethe $[13,14]$. Elle prévoit, pour des tensions inférieures à $1 \mathrm{MV}$, une dose critique proportionnelle à $\beta^{2}$ où $\beta$ représente le rapport de la vitesse des électrons et de la lumière. Cependant, l'expérience s'écarte quelquefois de cette loi, puisque Kobayashi et Ohara ont trouvé une proportionnalité en $\beta^{3}$ [11]. Au-delà de $1000 \mathrm{kV}$, la loi de Bethe prévoit un écart à la proportionnalité en $\beta^{2}$, mais les travaux expérimentaux sont pratiquement inexistants. Dupouy [15] trouve une augmentation de 1,5 de la dose critique de la thymine entre 500 et $3000 \mathrm{kV}$.

Dans cet article, nous exposons nos résultats expérimentaux obtenus avec les cristaux de polyéthylène ( 3 et 4$)$. Ces résultats sont ensuite comparés avec les prévisions théoriques de la loi de Bethe (\$5). 
Cependant le calcul du pouvoir d'arrêt par la formule de Bethe suppose que les atomes du polyéthylène sont indépendants les uns des autres : ils sont alors soumis à la même intensité d'électrons incidents (approximation de Born). Ce calcul est donc approprié dans le cas d'un matériau amorphe et mince. Nos cristaux sont effectivement très minces (maximum $20 \mathrm{~nm}$ ) et la diminution de l'intensité par absorption $\mathrm{y}$ est certainement négligeable. Mais qu'en est-il de l'influence de la cristallinité ?

Dans un cristal, les ondes ne sont pas réparties de façon homogène, à cause de phénomènes de résonance avec le réseau cristallin, qui se traduisent par une canalisation le long des plans du réseau [16]. On peut donc s'attendre à ce que la perte d'énergie dans un cristal diffère de celle d'un objet amorphe et varie en fonction de l'orientation.

Les paragraphes 6 à 8 sont consacrés à l'étude de la canalisation des ondes dans le polyéthylène et à l'évaluation de son influence sur le pouvoir d'arrêt. On montre que ce pouvoir d'arrêt corrigé s'ajuste mieux aux résultats expérimentaux entre 100 et $2500 \mathrm{kV}$.

\section{Conditions expérimentales.}

2.1 MONOCRISTAUX DE POLYÉTHYLÈNE. — Les cristaux ont été obtenus par la méthode d'autoensemencement [17] dans du tétrachloroéthylène. Il s'agit de Manolène dont les masses moyennes sont $M_{\mathrm{n}}=11000$ et $M_{\mathrm{w}}=90000$. Ils ont ensuite été déposés sur des grilles recouvertes d'un film de carbone, avec une concentration suffisamment faible pour qu'ils restent isolés. Les observations ont été effectuées avec un microscope électronique à transmission construit par le laboratoire, fonctionnant entre 1 et $3 \mathrm{MeV}$, ainsi yu avec un microscope à $100 \mathrm{kV}$.

\subsection{MeSURE DE LA DOSE REÇUE PAR L'ÉCHANTILLON.} - La dose $D$ s'exprime en Coulomb $/ \mathrm{m}^{2}$ ou en nombre d'électrons $/ \mathrm{nm}^{2}$. Elle est le produit du temps d'exposition au faisceau par l'intensité que nous avons maintenu constante. Sa valeur a été choisie suffisamment faible $\left(0,8 \mathrm{~A} / \mathrm{m}^{2}=5 \mathrm{e} / \mathrm{nm}^{2} . \mathrm{s}\right)$ afin que la durée de vie des échantillons soit compatible avec les manipulations. Aussi les fluctuations de l'intensité sont-elles fréquentes et introduisent une marge d'erreur importante dans la mesure, même si l'on élimine les mesures pour lesquelles un contrôle a montré que l'intensité à la fin de l'exposition était différente de l'intensité initiale.

2.3 DÉFINITION DE LA DOSE CRITIQUE. - Remarquons qu'on peut définir non pas une mais plusieurs doses critiques pour un même échantillon, relatives au caractère particulier qui nous intéresse dans l'échantillon : perte de masse, disparition d'une structure ou d'une surstructure, etc... Nous discuterons ici uniquement de la destruction de l'ordre cristallin, objectivée par la disparition complète des faisceaux diffractés.
Même avec cette définition restrictive, la notion reste imprécise puisque l'évaluation de la disparition des taches dépend $a$ priori de l'œil de l'observateur, de l'émulsion photographique utilisée, du mode de développement, du temps de pose, du réglage du microdensitomètre. On définit habituellement la dose critique $D_{\mathrm{c}}$ comme la dose $D_{\mathrm{f}}$ pour laquelle les taches de diffraction ont disparu $[10-12,14,15,18]$. Nous avons déjà discuté la subjectivité de cette méthode [19] et montré qu'elle était fiable si l'on s'en sert uniquement pour comparer des mesures faites sur le même appareil. D'autres auteurs ont proposé une méthode plus objective qui consiste à définir la dose $D_{\mathrm{c}}$ comme celle, $D_{\mathrm{e}}$, pour laquelle l'intensité $I$ du faisceau diffracté est égale à $37 \%$ de sa valeur initiale : $I=I_{0} / e[8,13,20]$. Elle n'est cependant applicable que dans le cas où $I$ subit une décroissance monotone.

Une autre difficulté provient du fait que la dose critique dépend du faisceau diffracté choisi [21]. Afin de préciser le choix d'une dose critique, nous avons enregistré l'évolution de l'intensité des faisceaux diffractés.

\section{Décroissance de l'intensité des faisceaux diffractés.}

Les diagrammes de diffraction ont été enregistrés (Fig. 1) à intervalles réguliers sur le film photographique, avec 2 temps de pose différents. La surface de l'échantillon contribuant au diagramme était d'environ $1 \mu \mathrm{m}^{2}$. La densité optique des taches a été

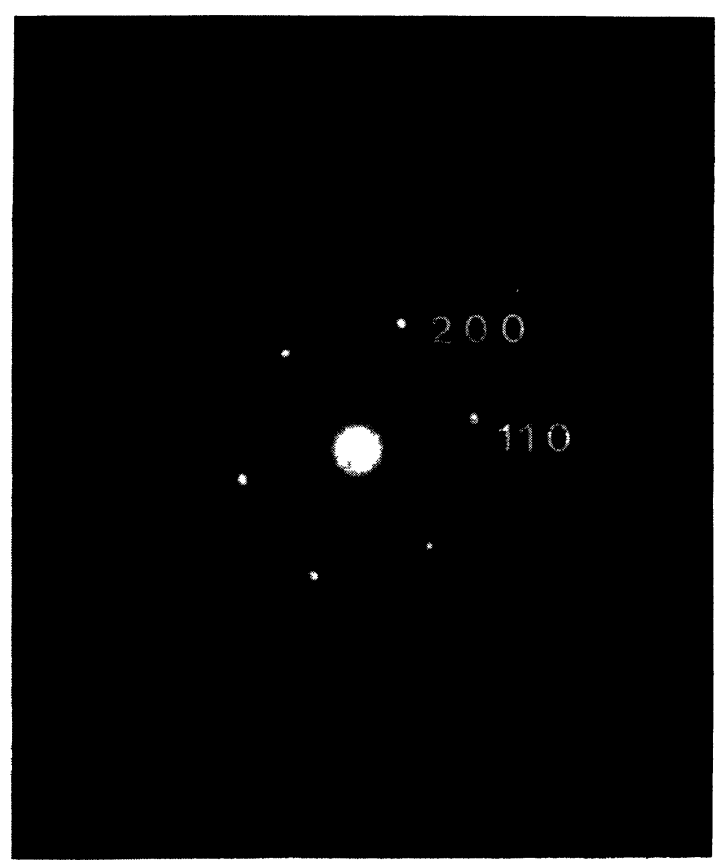

Fig. 1. - Diagramme de diffraction électronique d'un monocristal de polyéthylène, distance des plans $110: 8,9 \AA$; distance des plans $200: 9,88 \AA$.

[Electron diffraction pattern of a polyethylene single crystal, distance of 110 planes : $8.9 \AA$; distance of 200 planes : $9.88 \AA$ A.] 
mesurée à l'aide d'un microdensitomètre (CDC, type Vassy MD3 à platine). L'indexation des diagrammes a été faite d'après la structure parfaitement connue des cristaux de polyéthylène [22] (Fig. 2).

3. 1 Résultats. - Les 3 courbes de la figure 3 représentent l'évolution de l'intensité de 3 faisceaux différents, $100,1 \overline{10}$ et 200 . On n'a pas reporté les courbes pour les taches symétriques $(\overline{11} 0, \overline{1} 10$ et $\overline{200})$ qui sont identiques.

On constate que les courbes sont différentes l'une de l'autre pour un temps $t<t_{1}$, puis à partir de $t_{1}$, évoluent parallèlement et se rassemblent au temps $t_{\mathrm{c}}$. Ceci a lieu quelle que soit la tension utilisée.

3.2 Discussion : TEMPS CRITIQUe. - Comme la décroissance n'est pas monotone, il est impossible de définir un temps $t_{\mathrm{e}}$ pour lequel $I=I_{0} \times 0,37$. De plus un temps caractéristique défini à partir de $I_{0}$ dépendrait $\mathrm{du}$ faisceau choisi.

Par contre le temps $t_{\mathrm{c}}$ est commun aux 3 courbes et assez bien caractérisé si on le définit d'une façon plus objective comme le temps pour lequel l'intensité $I$ devient égale à celle du bruit de fond du film photographique, dans des conditions de pose et de développement bien définies [19]. C'est ce temps $t_{\mathrm{c}}$, correspondant à une dose $D_{c}$, que nous avons choisi pour caractériser la résistance du cristal aux électrons.

Remarquons qu'au temps $t_{\mathrm{c}}$, on a environ $I=I_{0} \times$ 0,05 et que $t_{1} \simeq 0,6 t_{\mathrm{c}}$. Les 2 définitions sont donc liées. $t_{1}$ est également le temps pour lequel les taches d'indices autres que $\{110\}$ et $\{200\}$ disparaissent. Il correspond à un temps utile pour lequel les images peuvent être considérées comme représentatives d'un objet non dégradé. Cette valeur $t_{1} / t_{\mathrm{c}}=0,6$ est en accord avec celle de Thomas et al. [20] déterminée à partir de la qualité des images en fond noir.

\subsection{INFLUENCE DE LA GÉOMÉTRIE DU CRISTAL. - Si} la plupart des auteurs ont constaté une décroissance monotone de l'intensité, quelques-uns ont, comme nous, enregistré des croissances initiales [18]. En image, nous avons observé corrélativement des changements importants de la position du cristal [5]. Bien que celui-ci soit en moyenne horizontal et que les molécules soient dirigées parallèlement à l'axe optique,

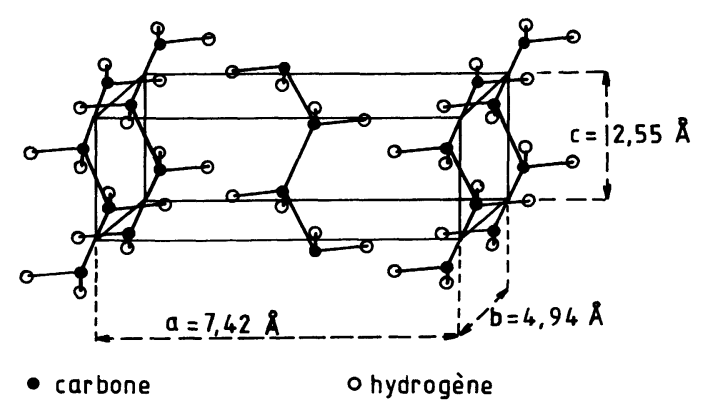

Fig. 2. - Structure de la maille du cristal de polyéthylène.

[Structure of the unit cell of the polyethylene single crystal.] la surface présente également des plis ou des ondulations qui s'affaissent ou basculent au cours de l'irradiation. Comme la diffraction correspond à une zone assez grande $\left(1 \mu \mathrm{m}^{2}\right.$ environ), elle subit les modifications d'orientation de cette zone. Des observations semblables ont été rapportées par d'autres auteurs dans la paraffine [23] et la L-valine [24].

Ce comportement est à l'origine d'une autre observation : si l'on constate sur la figure 3 et dans la plupart des cas que la dose $D_{c}$ est la même pour les 3 taches, il arrive cependant qu'une des taches se maintienne plus longtemps que les autres (soit une tache 200, soit une tache 110). Cela est probablement dû à une orientation locale particulière. A cause des plis et ondulation signalés plus haut, certaines parties de la zone contribuant à la diffraction se trouvent momentanément sous une incidence oblique et ne subissent pas la même irradiation à cause des effets de canalisation des électrons le long des colonnes atomiques.

Nous n'avons malheureusement pas pu préciser expérimentalement ce fait car la proportion des plis, leur orientation et leur évolution sont aléatoires. Aussi avons-nous simplement évalué par le calcul l'influence de la canalisation et de l'orientation de l'objet sur la dose critique (ci-dessous $\S 6$ à 8 ).

\section{Variation de la dose critique en fonction de la tension d'accélération.}

Les résultats des mesures de dose critique à la température ambiante, pour des tensions comprises entre 1 et 2,5 MV, sont rapportés sur la figure 4. Par comparaison, on y a également porté une mesure à $100 \mathrm{kV}$. Compte tenu des marges d'incertitudes, on peut noter que la dose est multipliée par 3 ou 4 en passant de 100 à $1000 \mathrm{kV}$. Pour des tensions plus élevées, elle reste la même.

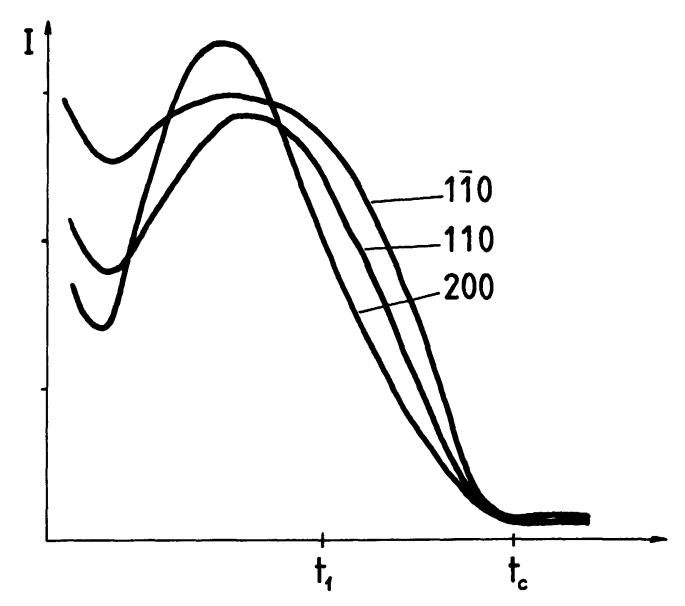

Fig. 3. - Evolution de l'intensité de 3 faisceaux diffractés.

[Evolution of the intensity of 3 diffracted beams.] 


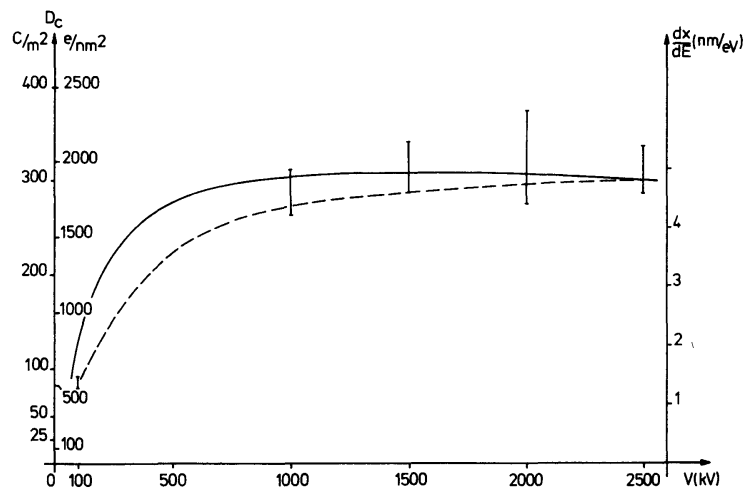

Fig. 4. - Barres verticales : résultats expérimentaux des mesures de la dose critique en fonction de la tension à température ambiante. Courbe en trait plein : inverse du pouvoir d'arrêt calculé par la formule de Bethe (coll. J. Ph. Perez). Courbe en pointillés proportionnelle à $\beta^{2}$.

[Vertical bars : experimental results of the measurement of the critical dose versus the voltage at room temperature. Continuous line curve : inverse of the stopping power calculated by the Bethe law (with. J. Ph. Perez). Dotted line curve proportional to $\beta^{2}$.]

\section{Comparaison avec la loi de Bethe.}

Nous avons calculé, en collaboration avec J. Ph. Perez, le pouvoir d'arrêt des électrons par le polyéthylène selon la loi de Bethe [25]. Elle nous fournit la valeur de la perte d'énergie par unité de longueur, dissipée lors des collisions inélastiques individuelles. Or, calculer le pouvoir d'arrêt par la formule de Bethe suppose que l'on se trouve dans certaines conditions que nous allons examiner :

- Les mécanismes par lesquels les électrons perdent leur énergie sont multiples. En premier, les chocs élastiques sur les noyaux de carbone et d'hydrogène sont 3 fois moins nombreux que les chocs inélastiques et l'énergie transférée au cours d'un choc est si faible en moyenne qu'ils peuvent être négligés. Parmi les processus inélastiques possibles, le rayonnement de freinage n'est pas mis en évidence pour des tensions inférieures à $3 \mathrm{MV}$. Quant aux plasmons, ce sont des oscillations collectives d'électrons quasi libres qui se produisent dans des conducteurs mais ont peu de chance d'apparaître dans des polymères. Il est généralement admis que les pics de pertes d'énergie de valeur voisine de $10 \mathrm{eV}$ dans les substances organiques proviennent de transition individuelle $\Pi \rightarrow \Pi^{*}$ ou $\sigma \rightarrow \sigma^{*}$ et non des plasmons [26].

En conséquence, les excitations individuelles sont prépondérantes et la formule de Bethe rend compte de toutes les excitations individuelles y compris des transitions $\Pi \rightarrow \Pi^{*}$ moyennant un ajustement du potentiel moyen d'ionisation $I$ du matériau [25]. Dans le cas du polyéthylène, $I=44 \mathrm{eV}$.

Pour des atomes indépendants recevant la même intensité incidente de 1 électron par seconde, le pouvoir d'arrêt s'exprime par la formule :

$$
\begin{aligned}
\frac{\mathrm{d} E}{\mathrm{~d} x} & =\frac{e^{4}}{8 \pi \varepsilon_{0} m c^{2}} N_{\mathrm{a}} \frac{Z}{\beta^{2}}\left[\log \frac{\left(\gamma^{2}-1\right)(\gamma-1) m^{2} c^{4}}{I^{2}}-\right. \\
& \left.-\left(\frac{2}{\gamma}-\frac{1}{\gamma^{2}}+1\right) \log 2+\frac{1}{8} \frac{(\gamma-1)^{2}}{\gamma^{2}}-\beta^{2}+1\right]
\end{aligned}
$$

où $e, m, v$, sont la charge, la masse et la vitesse de l'électron, $c$ la vitesse de la lumière, $\beta=v / c, \gamma=$ $\left(1-\beta^{2}\right)^{-1 / 2}$ et $N_{\mathrm{a}}$ est le nombre d'atomes de type a par unité de volume.

Si $E_{\mathrm{c}}$ désigne l'énergie totale transférée par unité de volume lorsque la cristallinité est détruite et $D_{c}$, la dose reçue par l'objet dans le même temps, alors :

$$
E_{\mathrm{c}}=\frac{\mathrm{d} E}{\mathrm{~d} x} \times D_{\mathrm{c}}
$$

ou

$$
D_{\mathrm{c}}=E_{\mathrm{c}} \frac{\mathrm{d} x}{\mathrm{~d} E}
$$

- Nous avons tracé la courbe $\mathrm{d} x / \mathrm{d} E$ sur la figure 4 en ajustant l'échelle de telle sorte que la courbe passe par les points expérimentaux de $D_{\mathrm{c}}$. Cela revient à dire qu'on a fixé un coefficient de proportionnalité $E$ entre l'échelle de $D_{c}$ et l'échelle de $\mathrm{d} x / \mathrm{d} E$. On constate qu'effectivement, cette courbe s'accorde bien avec les mesures de $D_{c}$. Les incertitudes de mesure assez grandes ne permettent pas de distinguer de petits écarts à cette loi.

L'accord entre les mesures de $D_{c}$ et le pouvoir d'arrêt $\mathrm{d} E / \mathrm{d} x$, montre que le coefficient de proportionnalité $E_{\mathrm{c}}$ est pratiquement constant et égal à $390 \mathrm{eV} / \mathrm{nm}^{3}$. En fait si l'on prend comme valeur $D$, la valeur moyenne de nos mesures expérimentales, à une tension donnée, les résultats varient légèrement : $368 \mathrm{eV} / \mathrm{nm}^{3}$ à $1000 \mathrm{kV}, 419 \mathrm{eV} / \mathrm{nm}^{3}$ à $2000 \mathrm{kV}$ et, un peu plus à l'écart, $247 \mathrm{eV} / \mathrm{nm}^{3}$ à $100 \mathrm{kV}$. Ces valeurs ne sont qu'indicatives car nous allons voir par la suite que la canalisation jouant un rôle non négligeable, il faut les corriger pour en tenir compte. Nous avons également comparé nos valeurs expérimentales $D_{c}$ en fonction d'une courbe en $\beta^{2}$ sur la figure 4 . On voit qu'elle n'est pas éloignée des valeurs expérimentales mais elle prévoit un accroissement continu de la dose qui n'est pas vérifié expérimentalement.

\section{Calcul de la densité des ondes électroniques dans le cristal.}

Nous nous proposons maintenant d'évaluer l'influence de la canalisation sur le pouvoir d'arrêt et le facteur $E_{\mathrm{c}}$ en fonction de la tension d'accélération des électrons, de l'épaisseur et de l'orientation de l'échantillon, à température ambiante.

Le traitement mathématique le plus rigoureux qui permette de calculer la perte d'énergie dans le cristal consisterait à considérer que ce cristal passe d'un état fondamental $|0\rangle$ à un état excité $|n\rangle$ tandis que 
l'électron passe d'un état initial $\left|\psi_{0}\right\rangle$ en dehors du cristal à un état $\left|\psi_{n}\right\rangle$ après le choc en ayant perdu une énergie $\Delta E_{n}$. La théorie des perturbations nous donnerait la probabilité $\sigma\left(E_{n}\right)$ de cette transition et la perte pourrait être calculée par :

$$
\Delta E=\sum_{n} \Delta E_{n} \cdot \sigma\left(E_{n}\right)
$$

Or $\psi_{n}$ est l'onde de l'électron à l'intérieur du cristal et se décompose en une infinité d'ondes de Bloch. Un tel calcul se révèle extrêmement complexe et peu justifié si l'on pense qu'il devra être confronté à des résultats expérimentaux entachés d'incertitudes de mesures assez importantes (cf. $\S 2.2$ ) et que ces résultats expérimentaux dépendent de processus moléculaires multiples et complexes introduisant de nombreux paramètres mal connus.

On ne peut affirmer qu'un modèle théorique est vrai uniquement parce qu'il s'ajuste bien aux données expérimentales. Quand le nombre des paramètres est grand, il est en effet possible de trouver plusieurs autres modèles différents qui s'ajustent également aux données par un choix adéquat des paramètres. Aussi, plutôt que de développer un calcul compliqué avec de nombreux paramètres qui ne pourrait pas être confronté avec l'expérience, il nous a paru plus judicieux et plus démonstratif de mettre en lumière comment la nature cristalline de notre objet modifiait la répartition des ondes électroniques. En d'autres termes, parmi les paramètres intervenant dans les pertes d'énergie, nous avons ici privilégié l'intensité incidente sur chaque atome qui nous paraît l'un des plus pertinents. Nous avons pu en déduire des conséquences essentielles sur le pouvoir d'arrêt, en fonction de la tension d'accélération des électrons, de l'épaisseur et de l'orientation du cristal.

Le calcul de l'inteñsité en chaque point du cristal a été mené selon le formalisme de la théorie dynamique. Les principes d'un tel calcul ont été exposés dans un article antérieur [27]. Dans le cas du polyéthylène, seuls 7 faisceaux ont été retenus pour le calcul, correspondant aux 7 faisceaux les plus intenses enregistrés expérimentalement sur un diagramme de diffraction (Fig. 1). Ce sont le faisceau central (000), 4 faisceaux diffractés (110) et 2 faisceaux (200). La diffusion de l'onde incidente est donc représentée par une matrice $7 \times 7$ et la valeur de l'onde $\psi$ en un point du cristal de coordonnées $(x, y, z)$ est donnée par :

$$
\begin{aligned}
\psi=\sum_{g} \sum_{i} C_{0 i} C_{g i} \exp \left(2 i \Pi \gamma_{i} t\right) \times & \\
& \times \exp 2 i \Pi(h x+k y+l z) .
\end{aligned}
$$

Son intensité est égale à $\psi^{*} \psi$.

Dans cette formule, $C_{g i}$ sont les coefficients de la matrice et $\gamma_{i}$ ses valeurs propres. Ils dépendent des coefficients de diffusion des atomes de carbone et d'hydrogène, ainsi que de leurs positions, de la tension et de l'orientation du cristal. $t$ est l'épaisseur. $(h, k, l)$ sont les indices des 7 faisceaux $g$. On a supposé que l'agitation thermique était nulle et que le cristal était parfait.

A la suite des collisions inélastiques, l'intensité du faisceau d'électrons diminue au fur et à mesure qu'il progresse dans le cristal, d'autant plus que l'épaisseur est grande. Dans le cas d'épaisseurs faibles, cette absorption est négligeable. Pour calculer l'influence éventuelle d'épaisseurs plus importantes, nous avons tenu compte de l'absorption en introduisant un potentiel complexe. Les $\gamma_{i}$ comportent alors une partie imaginaire.

La partie imaginaire du potentiel est prise égale au $1 / 10^{\mathrm{e}}$ du potentiel réel à $100 \mathrm{kV}$, et corrigé pour des tensions plus grandes [27].

La géométrie du cristal est décrite sur les figures 2 et 5. La maille élémentaire est orthorhombique, les chaînes moléculaires sont orientées parallèlement à l'axe c auquel est attribuée la coordonnée $z$. Aux axes a et b correspondent $x$ et $y$. A l'échelle macroscopique, l'axe c de la maille est perpendiculaire à la surface, qui contient donc les axes $\mathbf{a}$ et $\mathbf{b}$. L'inclinaison du faisceau est repéré par 2 angles $\left(\theta_{1}\right.$ et $\left.\theta_{2}\right)$ mesurés par rapport aux axes [110] et [200]. Le calcul a été fait pour 3 positions différentes :

(00), position symétrique, c'est-à-dire faisceau orienté parallèlement à c.

(11), en réflexion de Bragg par rapport aux plans 110.

(20), en réflexion de Bragg par rapport aux plans 200.

\section{Résultats des calculs : cartes de densité.}

7.1 INTENSITÉ À LA SURFACE DE SORTIE. - Pour une épaisseur donnée ( $z$ fixé), une tension et une orientation données, la variation de l'intensité en fonction de $x$ et $y$ peut être représentée par une carte de la face de sortie du cristal (Fig. 6). L'intensité n'y est pas homogène, mais passe par un maximum au voisinage des atomes de carbone, et ceci quelles que soient les conditions de tension, d'épaisseur ou d'orientation. C'est le phénomène de canalisation.

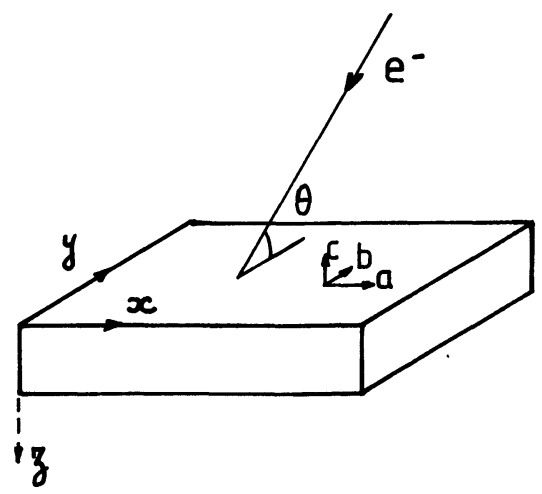

Fig. 5. - Géométrie d'un cristal de polyéthylène.

[Geometry of a polyethylene single crystal.] 


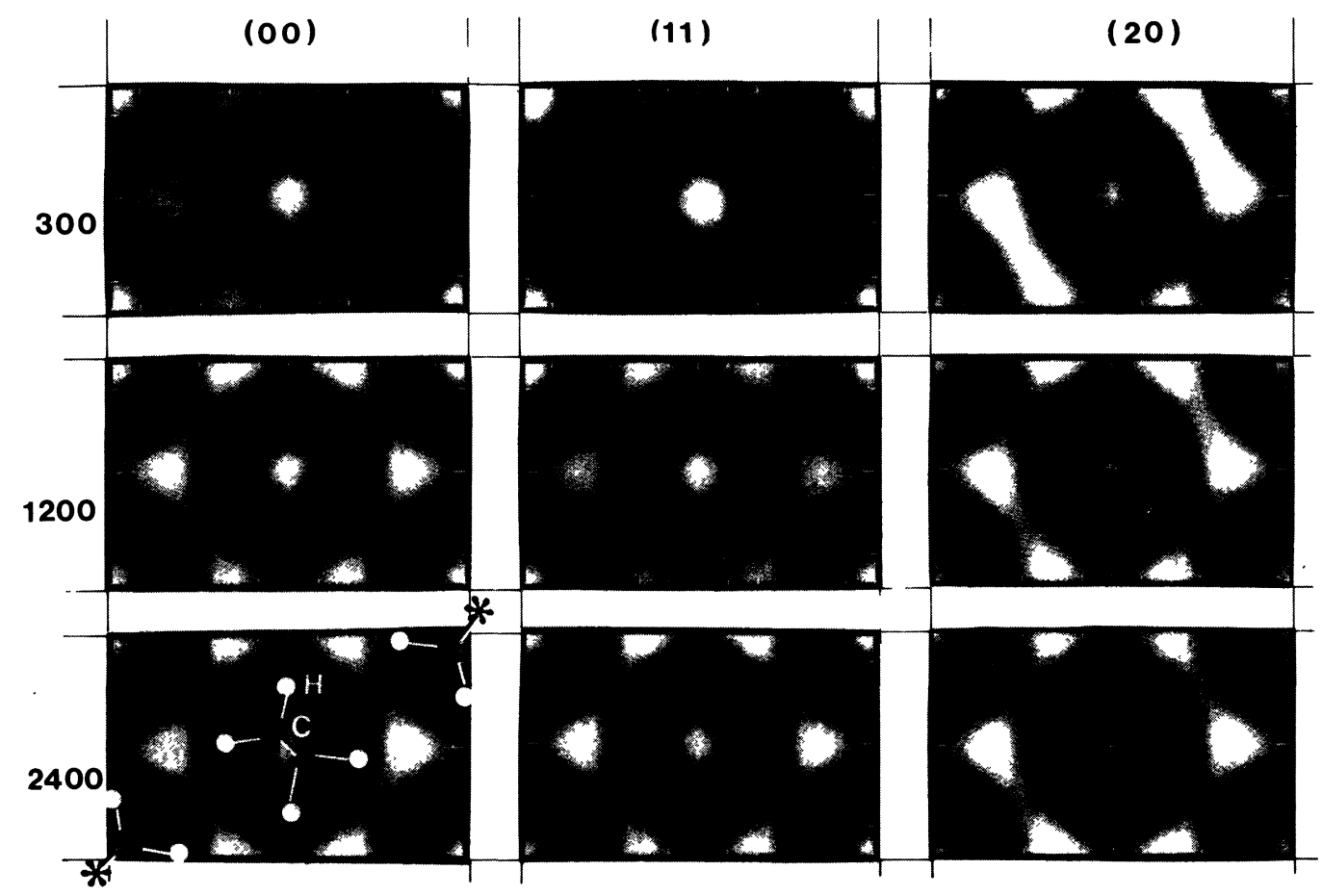

Fig. 6. - Carte de la densité électronique sur la face de sortie du cristal de $12 \mathrm{~nm}$ d'épaisseur ; a) à $300 \mathrm{kV}$, b) à $1200 \mathrm{kV}$, c) à $2400 \mathrm{kV}$. (00) faisceau parallèle à c, (11) faisceau en position de Bragg pour les plans (110), (20) faisceau en position de Bragg pour les plans (200).

[Map of the electronic density at the bottom surface of a $12 \mathrm{~nm}$ thick crystal ; a) at $300 \mathrm{kV}, \mathrm{b}$ ) at $1200 \mathrm{kV}$, c) at $2400 \mathrm{kV}$. (00) beam parallel to c, (11) beam in Bragg position with respect to the (110) planes, (20) beam in Bragg position with respect to the (200) planes.]

L'intensité incidente $I_{\mathrm{i}}$ a été arbitrairement fixée à 1. L'intensité $I$ au niveau d'un atome de carbone à la sortie atteint une valeur $1,5 I_{\mathrm{i}} \grave{a} 3 I_{\mathrm{i}}$ selon la tension et l'épaisseur. Il en résulte qu'au voisinage des atomes de carbone, les chocs inélastiques sont 1,5 à 3 fois plus nombreux que ne le suppose la formule de Bethe. En conséquence, le pouvoir d'arrêt est plus important dans un cristal que dans un matériau amorphe.

\subsection{INFLUENCE DE L'ÉPAISSEUR DE L'ÉCHANTILLON. -} Pour de faibles épaisseurs, c'est-à-dire environ jusqu'à $25 \mathrm{~nm}$ soit $100 c$ ( $c$ défini sur la Fig. 2) l'effet de l'absorption est négligeable. Pour des épaisseurs croissantes, l'intensité moyenne sur la surface reste la même, égale à $I_{i}=1$. Par contre l'amplitude entre le minimum et le maximum augmente (Fig. 7). Le maximum à l'emplacement de l'atome de carbone augmente de 1 à 2,5 pour des épaisseurs croissant de $c(2,5 \mathrm{~nm})$ à $100 \mathrm{c}$. C'est un effet typique de la canalisation : plus le «canal » est long et plus la résonance se manifeste avec une tendance des électrons à se tasser le long des colonnes atomiques et à déserter les espaces interatomiques.

Lorsque l'épaisseur augmente au-delà de $100 c$, ce phénomène est tempéré par l'absorption qui diminue l'intensité moyenne. La courbe de l'intensité le long de l'axe $y$, par exemple, s'écrase dans son ensemble.
Cela est particulièrement net pour des épaisseurs de l'ordre de $500 c(127 \mathrm{~nm})$ et au-delà, où l'absorption joue un rôle prépondérant.

Dans la suite de cet article, nous nous restreindrons à l'étude du cas de l'épaisseur réelle de notre cristal, soit environ $12 \mathrm{~nm}$, où, donc, le phénomène de canalisation est le plus manifeste.

\subsection{EFFET DE L'ORIENTATION DU CRISTAL PAR RAPPORT} AU FAISCEAU INCIDENT. - L'orientation ne modifie que légèrement les valeurs de l'intensité. C'est ce qui ressort des résultats des calculs effectués pour 3 orientations différentes : (00) faisceau parallèle à c, (11) faisceau en position de Bragg par rapport aux plans (110) et (20), en position de Bragg par rapport aux plans (200). Ces résultats sont reportés sur la courbe de la figure 8 le long de l'axe a de la face de sortie et imagés sur la figure 6 pour l'ensemble de la surface de sortie.

On remarque que l'intensité (11) est 1,2 fois plus forte que l'intensité (20) au niveau de l'atome de carbone.

7.4 EFFET DE LA TENSION D'ACCÉLÉRATION. - Dans les positions (00) et (11) l'augmentation de la tension entre $300 \mathrm{kV}$ et $3000 \mathrm{kV}$ a pour effet de diminuer l'intensité à l'emplacement du carbone d'un facteur 1,7 


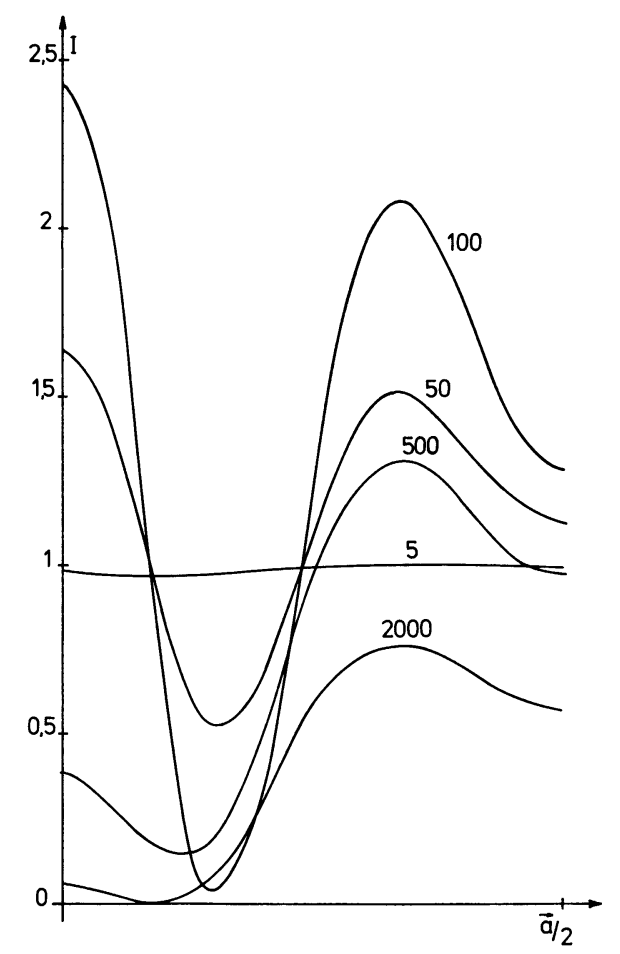

Fig. 7. - Densité électronique le long de l'axe a pour des épaisseurs variant entre $5 c$ et $2000 c, 1000 \mathrm{kV}$, position symétrique.

[Electronic density along the a axis for thicknesses between 5 and $2000 c ; 1000 \mathrm{kV}$; symmetrical position.]

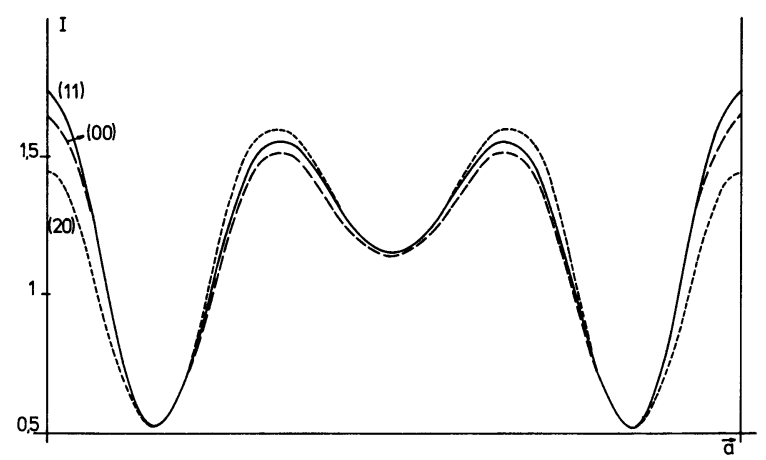

Fig. 8. - Densité électronique le long de l'axe a en fonction de l'orientation du faisceau incident. $1000 \mathrm{kV}$, épaisseur $12 \mathrm{~nm}$. (00) faisceau parallèle à c. (11) position de Bragg pour les plans (110). (20) position de Bragg pour les plans (200).

[Electronic density along the a axis for different orientations of the incident beam; $1000 \mathrm{kV}$; thickness $12 \mathrm{~nm}$; (00) beam parallel to $\mathrm{c}$; (11) Bragg position for (110) planes; (20) Bragg position for (200) planes.]

(cf. Fig. 9). En position (20), la canalisation est moins importante et la diminution n'est que de 1,17.

Vers les hautes tensions $(3000 \mathrm{kV})$, la différence entre les orientations s'estompent et l'intensité tend vers une valeur commune proche de 1,4 qui atteste que le phénomène de canalisation est encore bien présent (cf. aussi Fig. 6).

\section{Pouvoir d'arrêt des électrons dans un cristal.}

8.1 Méthode de CALCUL. - Nous avons calculé le pouvoir d'arrêt en supposant que chaque atome était soumis à une intensité incidente $I_{\mathrm{i}}=1(\S 5)$.

En réalité, l'intensité incidente n'est plus $I_{\mathrm{i}}$ uniformément dans le cristal mais $I(x, y, z)=\alpha(x, y, z) I_{\mathrm{i}}$ et nous avons vu au paragraphe précédent que $\alpha$ pouvait prendre des valeurs de l'ordre de 1,5 à 3 au voisinage des atomes de carbone, à la face de sortie du cristal.

Nous allons séparer le pouvoir d'arrêt en 2 termes :

$$
\left(\frac{\mathrm{d} E}{\mathrm{~d} x}\right)_{1}=\left(\frac{\mathrm{d} E}{\mathrm{~d} x}\right)_{\mathrm{c}}+\left(\frac{\mathrm{d} E}{\mathrm{~d} x}\right)_{\mathrm{v}} .
$$

Le premier est calculé au voisinage des atomes de carbone, c'est-à-dire grosso modo dans un rayon de 0,1 $\mathbf{a}=0,7 \AA$. Le deuxième dans l'espace vide entre les atomes (en négligeant les atomes d'hydrogène). Etant donné que la probabilité de choc $\sigma_{\mathrm{i}}$ est faible dans l'espace vide et que l'intensité $I$ y est aussi plus faible, on néglige la contribution de $\left(\frac{\mathrm{d} E}{\mathrm{~d} x}\right)_{\mathrm{v}}$ et il reste :

$$
\left(\frac{\mathrm{d} E}{\mathrm{~d} x}\right)_{1}=\left(\frac{\mathrm{d} E}{\mathrm{~d} x}\right)_{\mathrm{c}}=\frac{I_{\mathrm{c}}}{I_{\mathrm{i}}} \Sigma_{\mathrm{i}} \sigma_{\mathrm{i}} \Delta E_{\mathrm{i}}=\alpha_{\mathrm{c}}\left(\frac{\mathrm{d} E}{\mathrm{~d} x}\right)_{0}
$$

$\sigma_{i}$ est la section efficace (probabilité) pour un type de choc et $\Delta E_{\mathrm{i}}$ l'énergie transférée correspondante.

$\alpha_{c}$ désigne la valeur de $\alpha$ au voisinage de l'atome de carbone. Si sa valeur est $\alpha_{1}$ à la sortie du cristal, sa valeur moyenne à l'intérieur du cristal est $\alpha=\frac{1+\alpha_{1}}{2}$. Il en résulte que le pouvoir d'arrêt réel (aux approximations faites près), est :

$$
\left(\frac{\mathrm{d} E}{\mathrm{~d} x}\right)_{1}=\alpha\left(\frac{\mathrm{d} E}{\mathrm{~d} x}\right)_{0}
$$

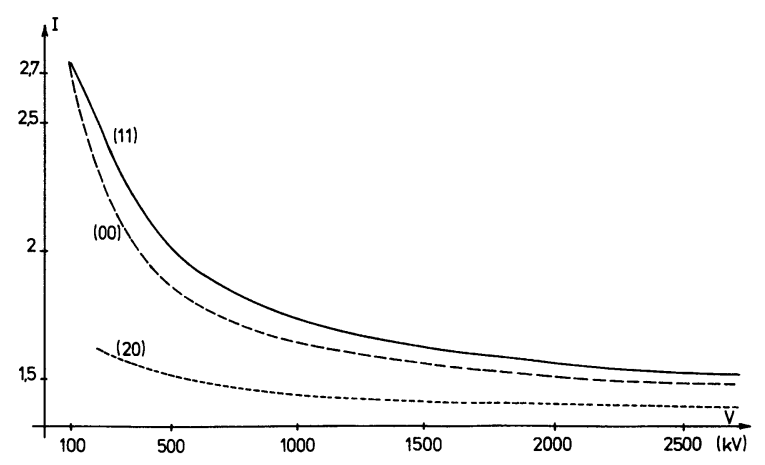

Fig. 9. - Densité électronique à l'emplacement de l'atome de carbone $(x=y=0)$ en fonction de la tension d'accélération des électrons pour 3 positions d'incidence. Epaisseur $12 \mathrm{~nm}$.

[Electronic density near the carbon atom $(x=y=0)$ versus the electron accelerating voltage for 3 incident beam positions; thickness $12 \mathrm{~nm}$.] 


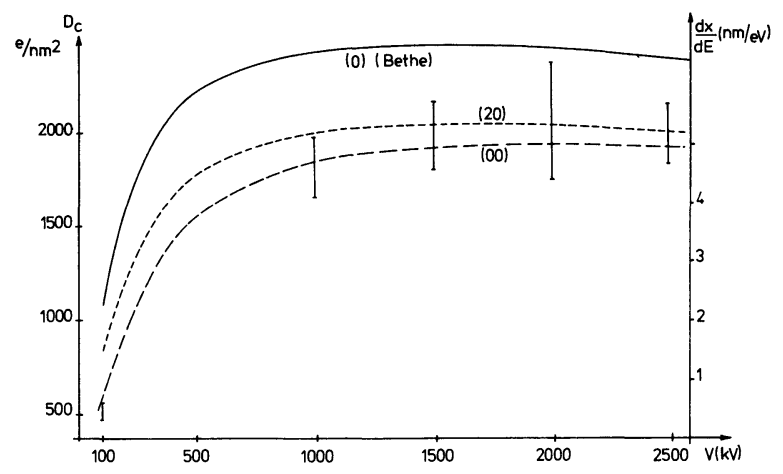

Fig. 10. - Barres verticales : résultats expérimentaux de la figure 3. Courbes : valeur de l'inverse du pouvoir d'arrêt. (0) sans canalisation (loi de Bethe), (00) canalisation en position symétrique, (20) position de Bragg pour les plans (200).

[Vertical bars : experimental results of figure 3. Curves : calculated value of the inverse of the stopping power. (0) without channelling (Bethe's law), (00) channelling in symmetrical position, (20) channelling in (200) Bragg position.]

$\alpha$ varie en fonction de la tension d'accélération, de l'épaisseur et de l'orientation du cristal entre 1,2 et 2 (§ 7, Figs. 7, 8, 9).

Sur la figure 10 , on a porté les valeurs calculées de l'inverse du pouvoir d'arrêt $(\mathrm{d} X / \mathrm{d} E)_{1}-$ qui nous sera par la suite plus utile que $(\mathrm{d} E / \mathrm{d} x)_{1}$ - c'est-à-dire le parcours correspondant à une perte d'énergie unité. Pour comparaison, la courbe $(\mathrm{d} x / \mathrm{d} E)_{0}$ obtenue avec $\alpha=1$ y a été reproduite, de même que les valeurs de $D_{\mathrm{c}}$. Le facteur de proportionnalité $E$, entre les deux échelles $D_{\mathrm{c}}$ et $\mathrm{d} x / \mathrm{d} E$ de la figure 10 , a été choisie différemment de celui de la figure 4, afin d'ajuster la courbe (00) aux résultats expérimentaux.

8. 2 Résultats. - Les résultats de la figure 10 traduisent l'influence de la canalisation sur le pouvoir d'arrêt. Par rapport à l'objet amorphe, la canalisation se manifeste par une augmentation du pouvoir d'arrêt (facteur $\alpha$ ) de l'ordre de 1,2 à 2 selon la tension et l'orientation, pour une épaisseur de $12 \mathrm{~nm}$. Cette augmentation est plus marquée aux basses tensions, de même que dans la position symétrique (00) et (11). La différence entre les orientations est cependant trop faible pour être mise en évidence par l'expérience.

Le maximum de la courbe de Bethe se situe à $1200 \mathrm{kV}$. Après correction, il se déplace à 1500 ou
$2000 \mathrm{kV}$ mais il est si minime que la courbe est quasiment horizontale de 1500 à $3000 \mathrm{kV}$.

La courbe de Bethe prévoyait une augmentation de la dose critique de 2,25 lorsque la tension croît de 100 à $1500 \mathrm{kV}$. Après correction due à la canalisation, ce facteur est augmenté à 2,4 en position (20) et 3,3 en position (00). Ceci est plus en accord avec nos mesures (facteur égal à 3). Autrement dit, la courbe corrigée s'accorde mieux avec la courbe expérimentale à $100 \mathrm{kV}$.

L'énergie critique doit être modifiée. La figure 10 montre que le meilleur ajustement de la courbe $\mathrm{d} x / \mathrm{d} E$ est obtenu pour un facteur de proportionnalité de $476 \mathrm{eV} / \mathrm{nm}^{3}$ (au lieu de 390 sans canalisation). Si l'on calcule le facteur $E_{\mathrm{c}}$ à partir des valeurs moyennes de $D_{\mathrm{c}}$ à chaque tension, on a

$$
E_{\mathrm{c}}=D_{\mathrm{c}}\left(\frac{\mathrm{d} E}{\mathrm{~d} x}\right)_{1}=D_{\mathrm{c}} \alpha\left(\frac{\mathrm{d} E}{\mathrm{~d} x}\right)_{0}=\alpha E_{\mathrm{c} 0}
$$

et l'on obtient des valeurs qui varient peu : $457 \mathrm{eV} / \mathrm{nm}^{3}$ à $100 \mathrm{kV}, 485$ à $1000 \mathrm{kV}, 523$ à $2000 \mathrm{kV}$.

\section{Conclusion.}

Nos mesures expérimentales ont montré que l'augmentation de la durée de vie des cristaux de polyéthylène à haute tension suit approximativement la loi de Bethe, et s'accorde mieux avec un pouvoir d'arrêt corrigé en tenant compte de la canalisation, essentiellement à basse tension où la canalisation est plus forte. Si la durée de vie augmente d'un facteur 3 lorsque la tension d'accélération passe de 0,1 à $1 \mathrm{MV}$, elle reste sensiblement constante au-delà de $1 \mathrm{MV}$.

L'étude théorique de la canalisation montre que celle-ci augmente avec l'épaisseur de l'objet, diminue avec la tension, est plus sensible dans certaines orientations. L'influence de l'orientation reste cependant en deçà des marges d'erreur expérimentales.

L'énergie critique déposée dans le cristal pour aboutir à la destruction est évaluée à environ 460 à $520 \mathrm{eV} / \mathrm{nm}^{3}$ à la température ambiante.

\section{Remerciements.}

A. Boudet tient à remercier J. Ph. Perez, A. Lannes et B. Jouffrey pour leurs suggestions ainsi que les techniciens du microscope de $3 \mathrm{MV}$, MM. Jouret, Bilotte, Sopena, Salles pour leur aide expérimentale. 


\section{Bibliographie}

[1] Agar, A. W., Frank, F. C. et Keller, A., Philos. Mag. 4 (1959) 37.

[2] GrubB, D. T., J. Mater. Sci. 9 (1974) 1715.

[3] BoudeT, A. et KubIN, L. P., in Electron Microscopy 1978, Ninth. Intern. Congr. Electron. Microsc., ed. J. M. Sturgess (Microscopical Society of Canada, Toronto) 1978, vol. 1, p. 498.

[4] Idem, in Electron Microscopy 1980, Seventh Eur. Cong. Elect. Microsc., ed. P. Brederoo and G. Broom (North Holland, Amsterdam) 1980, vol. 1, p. 434.

[5] Boudet, A., J. Mater. Sci. 19 (1984) 2989.

[6] BoudeT, A. et KUBIN, L. P., Ultramicroscopy 9 (1982) 409.

[7] Glaeser, R. M., in Electron Microscopy and Microbeam Analysis, ed. Siegel et Beaman (Wiley and Sons) 1975, p. 205.

[8] IsAaCsON, M.S., in Principles and Techniques of Electron Microscopy, ed. Hayat (Van Nostrand Reinhold Company) 1977 , p. 1.

[9] BoudeT, A., partie II.

[10] Kobayashi, K. et SaKaOKu, K., Bull. Instrum. Res. 42 (1964) 473.

[11] Kobayashi, K. et Ohara, M., Sixth inter. congr. elect. microsc. (Maruzen, Tokyo) 1966, vol. 1, p. 579.

[12] Thomas, L. E., HumphreYs, C. J., DuFF, W. R. et GrUBB, D. T., Rad. Effects 3 (1970) 89.

[13] REIMER, L., in Electron Microscopy and Microbeam Analysis, ed. Siegel et Beaman (Wiley and Sons) 1975, p. 231.
[14] Glaeser, R. M., J. Ultrastr. Res. 36 (1971) 466.

[15] DupouY, G., High voltage electron microscopy, proceeding of the third intern. conf. ed. P. R. Swann, G. J. Humphreys, M. J. Goringe (Academic Press, London) 1974, p. 441.

[16] Hirsch, P., Howie, A., Nicholson, R. B., Pashley, D. W. et Whelan, M. J., Electron Microscopy of Thin Crystals (R. E. Krieger, Malabar, Florida) 1977, p. 215.

[17] Wunderlich, B., Macromolecular Phys. (Academic Press, New York, London).

[18] Grubb, D. T. et Groves, G. W., Philos. Mag. 24 (1971) 815.

[19] BoudeT, A., Thèse de doctorat, Toulouse 1981.

[20] Thomas, E. L., Sass, S. L. et Kramer, E. J., Philos. Mag. 30 (1974) 335.

[21] Read, R. T. et Young, R. J., J. Mater. Sci. 19 (1984) 327.

[22] Swan, P. R., J. Polymer Sci. 56 (1962) 409.

[23] WADE, R. H., Ultramicroscopy 14 (1984) 265.

[24] KnapeK, E., FormaneK, H., Lefranc, G. et Dietrich, I., Ultramicroscopy 14 (1984) 253.

[25] Perez, J. Ph., J. Physique 44 (1983) 699.

[26] IsaAcson, M., Johnson, D. et Crewe, A. V., Rad. Res. 55 (1973) 205.

[27] Roucau, C. et Ayroles, R., Philos. Mag. 31 (1975) 387. 